\title{
EL COLEGIO SANTA ISABEL DE HUNGRÍA EN SU CINCUENTENARIO: DE UNA INSERCIÓN EN COMUNA SEMI-RURAL HA PASADO A COMUNA URBANA ${ }^{1}$
}

\author{
Dr. Jaime Caiceo Escudero²
}

\begin{abstract}
RESUMO
Durante la década del 40 del siglo pasado (1944), la Madre Teresa Ortúzar Ovalle, en conjunto con Mons. Bernardino Berríos Gaínza, Obispo de San Felipe de Aconcagua, Chile, fundaron la Congregación Religiosa Hermanas Franciscanas Cooperadoras Parroquiales, teniendo como una de sus misiones, la educación de niños y niñas provenientes de sectores más vulnerables. Fue así como la propia Madre Teresa fundó en la Comuna de La Cisterna en Santiago de Chile la Escuela ${ }^{3}$ Particular Santa Isabel de Hungría en 1961; con el correr de los años se transformó en el actual Colegio ${ }^{4}$ del mismo nombre. La Comuna de La Cisterna en esa época era una comuna semi-rural en que había casas con parcelas y/o quintas; sus habitantes eran de clase media y clase media baja; por el desarrollo urbanístico, la comuna en cuestión perdió sus parcelas y quintas y se fue transformando en un lugar eminentemente urbano, manteniendo el mismo estrato social. Durante el año 2011, el establecimiento cumplió 50 años. El objetivo del presente artículo es reconstituir los principales hitos históricos del Colegio Santa Isabel de Hungría, indicar su misión institucional y si su desarrollo ha respondido a la visión de la fundadora. De una escuela primaria con muy pocos alumnos se ha pasado a un establecimiento educacional que entrega tres niveles diferentes de enseñanza (educación pre-escolar, enseñanza básica y enseñanza media) a 1.170 alumnos y alumnas. La metodología utilizada es la propia de los estudios históricos, es decir, se ha recurrido a fuentes primarias y secundarias, como análisis de documentos, archivos fotográficos y especialmente entrevistas (historia oral).
\end{abstract}

Palavras-chave: Educación religiosa; Escuela semi-rural; Colegio urbano; Historia educacional.

\section{SANTA ISABEL DE HUNGRIA SCHOOL IN ITS FIFTIETH ANNIVERSARY: FROM THE INSERTION IN A SEMI-RURAL AREA TO AN URBAN COUNTY}

\begin{abstract}
During the 40's of last century (1944), Mother Teresa Ortuzar Ovalle with Bishop Bernardino Berrios Gainza, Bishop of San Felipe de Aconcagua, Chile, founded the Religious Congregation of Franciscan Cooperating Parish Sisters. One of their missions was the education of the most vulnerable children; because of this fact, Mother Teresa herself founded Santa Isabel de Hungria School ${ }^{5}$ in La Cisterna, Santiago, Chile, in 1961. With the passing of the years this institution was transformed into the present high school ${ }^{6}$ with the same name. At that time, La Cisterna County was semi-rural full of land lots or isolated houses. Its inhabitants were middle and lower-middle class; because of the urban development, this area lost its land lots and became an urban place keeping the same social stratum. In 2011, the institution will be 50 years. The aim of this paper is to reconstruct the historical milestones of Santa Isabel de Hungria School and indicate their institutional mission analyzing if its development has responded to the vision of its founder. This school started as a primary school for a limited number of students and nowadays, it has turned into
\end{abstract}


a co-educational institution that provides three different levels of education (preschool, primary and secondary education) for 1170 students. The methodology used is typical of historical studies, that is, primary and secondary sources, such as analysis of documents, photos, archives and specially interviews (Oral History).

Keywords: Religious education; Semi-rural school; Urban College; History education.

\section{Introducción}

En la teoría organizacional se afirma que es más difícil mantener una institución en el tiempo que fundarla. Por lo mismo, con motivo del cincuentenario de la fundación del Colegio Santa Isabel de Hungría por parte de la Madre Teresa Ortúzar Ovalle (1961) en la comuna de La Cisterna de Santiago de Chile -semi-rural en esa época-, parece relevante detenerse en el tiempo y analizar el desarrollo histórico de este establecimiento educacional.

Antes de morir, la Fundadora señaló: "Yo me iré, pero ustedes quedarán para sostener este edificio, levantarlo y hacerlo progresar... Y si, es obra de Dios, no perecerá" (ORTÚZAR in MADARIAGA, 1993, p. 12). Al llegar a su primer cincuentenario de existencia se puede comprobar en forma empírica que efectivamente esta obra es de Dios, pues no ha perecido sino que, al contrario, algo que surgió como una aventura sin mayores recursos, recibió el impulso inicial de la Madre Teresa, pero luego el esforzado trabajo de las Hermanas, especialmente de la primera Directora, la Hna. Paula Hernández (1961-1966) junto a las Hermanas Martina Páez, Eufrosina Moreno y Myriam Obreque, entre otras, dieron una muestra de entrega total a la obra; esa acción significó que la comunidad, especialmente los padres y apoderados se volcaran a apoyarlas. Este esfuerzo significativo de religiosas y apoderados fue cavando las bases de una institución que con el tiempo se fue solidificando; esa forma inicial de trabajo significó partir construyendo en roca sólida.

Este artículo es el resultado de una investigación que se realizó en dos etapas: período 2002-2003 y período 2008-2009; el trabajo fue coordinado y dirigido, como investigador principal, por el autor de este trabajo y la participación de algunos ayudantes. Se realizó con la metodología científica propia de los estudios históricos, revisando archivos, documentos legales, testimonios fotográficos, infraestructura y entrevistando a testigos presenciales, es decir, se recurrió a fuentes primarias; también se utilizaron fuentes secundarias, como textos y otros documentos que ayudaron a contextualizar el momento histórico en que se fundó el Colegio Santa Isabel de Hungría. Los testimonios personales expresados en las entrevistas son la parte más valiosa de este trabajo desde la perspectiva de la Historia Oral.

\section{Fundación de la Congregación Religiosa Hermanas Franciscanas Cooperadoras Parroquiales}

Corría la década del 40 del siglo pasado cuando la Providencia logró que dos seres humanos, amantes de su Iglesia y de los principios evangélicos llevados a la práctica bajo el carisma franciscano, juntaran sus anhelos y esfuerzos para que surgiera una nueva congregación religiosa católica; ellos fueron la Madre Teresa Ortúzar Ovalle (1882-1969) y Monseñor Roberto Bernardino Berríos Gaínza (1885-1975), Obispo de San Felipe -ciudad distante a $90 \mathrm{kms}$. de la capital-. Ambos unieron su visión y deseo de dotar al obispado de una institución que apoyara el servicio de la Iglesia en la Diócesis. 
Tras cuatro años de prueba, el Pastor le concedió el decreto de fundación, registrado en la página 128 del Libro de Decretos del Obispado. Así se fundó la Pía Unión de las Cooperadoras Parroquiales de la Inmaculada Concepción, Terceras Franciscanas con fecha 8 de diciembre de 1944. Textualmente se señaló:

Venimos a fundar en nuestra Diócesis la Pía Unión de las Cooperadoras Parroquiales de la Inmaculada Concepción, Terceras Franciscanas...en el año de 1944 la Diócesis de San Felipe de Aconcagua, formando de esa manera la nueva Congregación denominada: 'Hermanas Franciscanas Cooperadoras Parroquiales'.

La nueva institución se preocupó de las necesidades de las parroquias; para cumplir adecuadamente tal labor, las Hermanas -aunque mujeres piadosas- tenían que recibir una formación espiritual; para ello tomaron a San Francisco de Asís como modelo de Cristo, pobre y humilde. De esta forma, la Madre Teresa lograba lo que tanto anhelaba y que había realizado dos intentos anteriores, tanto en el norte de Chile como en Lomas de Zamora en Buenos Aires.

Algunos testimonios nos revelan la personalidad de la Madre Teresa. Su vida de adolescente transcurrió dedicada al estudio, oración y excursiones al aire libre. En esos años forjó una recia voluntad y carácter firme. "La meditación diaria, la lectura espiritual, largas horas a los pies de Jesús Sacramentado, penitencias por medio de continuos ayunos y cilicios que apretaban su cuerpo hasta sangrar formaron una personalidad profundamente religiosa dedicada toda al amor de Dios" (MADARIAGA, 1993, p. 1).

La Hna. Eufrosina valora su voluntad por lograr lo que se proponía, partiendo de los escasos recursos que ella podía administrar. Así lo recuerda: “... lo que se proponía luchaba y luchaba. Como religiosa, un corazón muy grande de Madre, una persona humilde con toda su inteligencia, muy pertinente, muy sacrificada, de mucha oración, porque ella tuvo su formación en las carmelitas" (MORENO, 2002).

La misma Hermana prosigue al señalar que tenía un corazón tan inmenso que decía "el día que yo me muera que no falte una cosa, no falte la misericordia" (MORENO, 2002).

La Hna. Martina Páez recuerda con mucho cariño su acogida y capacidad de escucharla; la consideraba toda una dama y la describe como “... una mujer extraordinaria, su sola figura cautivaba, muy dedicada y creo de tipo mística, de oración profunda, de una caridad extraordinaria, de un amor inmenso al Señor..." (PÁEZ, 2002).

Respecto a la fundación de la Congregación por parte de Mons. Berríos, la Hna. Mercedes, ya fallecida, expresaba en sus recuerdos:

[...] él vio que Nuestra Madre quería juntar juventud para formarla y Monseñor tenía sus ideas; así unen estas ideas. Él dijo que estaba bien y las apoyó, venía cada quince días a darles formación, a darles instrucciones y él quería que fuéramos de vida activa, que estuviésemos con la gente, que los visitáramos y él, como era un misionero todo el año, visitaba sus Parroquias hasta el Choapa. Llegaba a toda su Diócesis, a sus Iglesias, constatando que estaban muy abandonadas, buscaba las soluciones (MUÑOZ, 2002).

\section{Fundación del Colegio Santa Isabel de Hungría}


Después de la fundación de la Congregación Religiosa Hermanas Franciscanas Cooperadoras Parroquiales en la ciudad de Los Andes -distante 15 kms. de San Felipe-, en 1944, quedando dependientes de la Diócesis de San Felipe, su radio de acción y labor se centró en la zona y alrededores, realizando un trabajo sistemático bajo el carisma franciscano: oración, contemplación, espíritu de sacrificio, amor a los pobres y fiel cumplimiento del Evangelio. Como Hermanas Cooperadoras Parroquiales, su labor directa se orientó a trabajar en las parroquias cercanas. Una tarea que se derivó de esta relación estrecha con dichas parroquias implicaba la incursión de las Hermanas en las escuelas parroquiales. La Hermana Eufrosina expresa esta vivencia de la siguiente manera:

Eran escuelas parroquiales, no eran propias de nosotras, pero prácticamente eso era lo que nos habían legado nuestros fundadores, que nosotros trabajáramos en escuelas parroquiales. Ese era el servicio y si en las escuelas parroquiales terminaba el acuerdo con el Párroco, entonces nos retirábamos de ese lugar o lo dictaminaba el Obispo de la Diócesis. Las Hermanas se retiraban y se trabajaba hasta ese momento, esperando otro lugar que nos acogiera (MORENO, 2002).

Las Hermanas Franciscanas Cooperadoras Parroquiales lograron establecer comunidades en Los Andes, Salamanca y Santiago, manteniendo su sede principal en Los Andes con el nombre de Casa de Santa María de los Ángeles. El empeño demostrado por ellas fue fuente de la creación de una escuela propia, la Escuela Santa Clara en Los Andes y La Escuela Santa Isabel de Hungría en la comuna de La Cisterna en Santiago.

Su llegada a La Cisterna -comuna semi-rural de Santiago de Chile-, a fines de los 50 del siglo pasado, para perfeccionar a las Hermanas en educación, permitió que ellas se incorporaran a la Parroquia San Juan Bosco, a cargo de los sacerdotes salesianos, ubicada en Paradero 22 1/2 de la Gran Avenida José Miguel Carrera. La residencia de las Hermanas en esa comuna y su colaboración en la Parroquia, posibilitó que el servicio y trabajo de la Congregación también echara raíces en ese lugar.

Fue así, como a través de la Parroquia se realizó una linda labor en una pequeña escuela de la población de San Ramón, zona habitada por gente pobre y humilde, en algunos casos de no tan buen vivir. La Hermana Rosario recuerda que era un Colegio para niñas humildes y destaca: “... era admirable, iban impecables, limpiecitas. Entonces nosotras llegábamos a ellos para formar a las niñas, para formar a los padres y para formar a los jóvenes de esos apoderados..." (VALDIVIA, 2002).

Es decir, ellas se esmeraban por atraer a todo el grupo familiar, mediante acciones deportivas como el ping-pong o el football. Dichas actividades se organizaban los días sábados, teniendo participación directa las mismas Hermanas en las competencias, llegando incluso a enfrentarse con equipos formados por apoderados y jóvenes.

El trabajo realizado daba sus frutos en esas personas, ya que las sacaba de la miseria y de la ignorancia. Si era necesario, las Hermanas reunían alimentos, ropas y otros elementos de primera necesidad e iban en ayuda a dar solución a las carencias que presentaban algunas de esas familias, pero esencialmente se pretendía enseñar el respeto a los adultos para proyectarlo en los niños y los jóvenes.

Considerando estas acciones, se dio el momento en que convergieron dos iniciativas para hacer realidad la creación de un establecimiento que diera educación a los niños y jóvenes de ese sector. Fue así como la visión de la Madre Fundadora, Madre Teresa Ortúzar, por dar una formación basada en sólidos principios y valores del cristianismo bajo el carisma 
franciscano, se enlazó con la necesidad palpada y manifestada por el Cura Párroco del lugar, Padre Francisco Andriguetti, sacerdote salesiano.

El sector que rodeaba las casas de las Hermanas recibía el nombre de Bungalows Santa Anselma; entre ellos y la Panamericana Sur sólo había predios agrícolas o sitios eriazos. En el sector había dos colegios particulares y pagados: San Juan Bosco y Madre Cecilia Lazzeri. A estos establecimientos no tenían acceso todos los niños, porque algunos vecinos eran muy humildes y eran gente que necesitaba este servicio. Marisa Cerda, una de sus primeras alumnas y hoy apoderada, señala al respecto:

[...] Éramos todas gente humilde, no teníamos acceso, aquí los únicos Colegios católicos era Don Bosco y Madre Cecilia Lazzeri, pero eran colegios pagados; entonces la Hermana Teresa se instaló aquí, porque quería darle educación a los niños pobres... La casa de nosotros estaba en calle Ibérica, era la última casa, para dentro había zarzamoras, nosotros íbamos a sacar yuyos. Eso indica que éramos pobres, el nuevo colegio estaría al alcance de nosotros; las familias eran numerosas, con varios hijos (CERDA, 2008).

Estas familias habían llegado al sector de La Cisterna porque los sitios se vendían a un precio módico, si bien ir a Santiago significaba una hora u hora y media de camino. Las Hermanas, al conocer las condiciones del lugar y el empeño de esas familias por echar raíces en él, construir sus casas por etapas y el interés por educar a sus hijos, posibilitó establecer un nuevo colegio, con carácter de particular, pero gratuito para los hijos de gente trabajadora del sector.

Antes de su creación, comenzó a funcionar en la Casa Belén y en la Casa Santa Isabel de Hungría un Kinder y una Primera Preparatoria a cargo de las Hermanas Matilde Parada y Aída Bustamante. De este hecho es testigo directo Marisa Cerda, como una de las pequeñitas que asistieron a ese curso. El lugar se organizó como un jardín chico que aglutinaba a unos pocos niños del sector, unos cuatro o cinco alumnos, no más, a los cuales era fácil enseñar y recuerda sobre ello: “... Éramos un grupo pequeño de niños de por aquí, íbamos a la Casa de ellas, a jugar, algunos no sabíamos leer y ellas nos enseñaban. ..." (CERDA, 2008). De igual modo, plantea que el hecho de ser vecinos de las Hermanas, la relación con ellas era cercana y el hecho de ir a la casa de ellas se puede comparar como la visita a casa de unas tías o familiar.

La hora de ingreso era a las ocho de la mañana. A la hora de almuerzo había dos alternativas, quedarse a almorzar o bien ir a almorzar a sus casas. Terminada la hora de almuerzo, se regresaba al jardín entre las cuatro o cuatro y media de la tarde, hasta completar la jornada en la tarde, a las seis.

En este período, la Madre Teresa Ortúzar estaba preocupada de adquirir un terreno que pudiese cobijar a la futura escuela, mientras la Casa Belén cumplía un papel importante recibiendo a los niños que realizaban el Kinder. Fue así como comenzó a comprar diferentes sitios en los cuales se han construido las dependencias del colegio Santa Isabel de Hungría; las adquisiciones se fueron haciendo paulatinamente, desde la esquina de la calle Santa Anselma con San Juan Bosco hasta la calle Brisas del Maipo, en 5 lotes, gracias a la cooperación de los Padres y Apoderados.

Finalmente, el 13 de noviembre de 1961, a través del Decreto $N^{\circ} 17.493$, se creó la Escuela Particular $N^{\circ} 447$ Santa Isabel de Hungría y el Ministerio de Educación Pública reconoció a las Hermanas como Cooperadoras de la Función Educacional del Estado. En ese año, también se concretó el traslado de la Escuela a su domicilio actual, calle Santa Anselma 
$\mathrm{N}^{\circ}$ 0240, tras la adquisición del primer terreno terreno por la Madre Fundadora, y se designó a la Hermana Paula Hernández como su primera Directora. En su cometido la acompañaron las Hermanas Eufrosina Moreno, Myriam Obreque, Martina Páez y Gladys Lagos.

Los cursos creados en la Casa Santa Isabel de Hungría llegaron a su nuevo domicilio desde Kinder a Cuarta Preparatoria. La nueva escuela presentaba dos salas y una oficina en una construcción modesta que existía en el terreno recién adquirido por las Hermanas. Para tener un lugar de juego para los niños o patio, hubo que despejar un sector posterior de la casa, cubierto de piedras. Se hizo necesario emparejarlo, uniendo sus fuerzas las Hermanas y los Padres y Apoderados.

La necesidad de habilitar el lugar, aunque modesto, revela el interés, esfuerzo y constancia de las Hermanas, de los primeros apoderados y del Padre Andriguetti, s.d.b.; el apoyo moral era esencial para dar unidad al grupo y concretar la tarea emprendida. La modesta infraestructura se demostraba en la falta de mobiliario para los alumnos, siendo necesario que los padres y apoderados llevasen sillas para que sus hijos o hijas pudiesen acomodarse en las salas de clases.

Entre sus recuerdos, Tatiana Yuffer, ex postulante de la Congregación y profesora del establecimiento, señala que la autorización dada por el Ministerio significó habilitar la Escuela, construyendo algunas salas, en las cuales se acomodaron dos cursos, ya sea primero y segundo, tercero y cuarto y así sucesivamente. Lo expresa de la siguiente manera:

En la sala se instalaba un pizarrón y colocaban desde la mitad de la sala las sillas mirando hacia el pizarrón, y luego se ponía un segundo pizarrón en el lado opuesto y se ponían las sillas mirando a éste y se colocaban los niños del otro curso. Así se inició primero y segundo, después tercero y cuarto. Y al año siguiente llegaron a tener quinto y sexto en las mismas condiciones, los niños separados en una misma sala (YUFFER, 2008).

De esta forma, se respondía a la aspiración de entregar una enseñanza gratuita que permitiera formar hombres y mujeres para el bien y para la patria. En otras palabras, “... constructores de valores intachables, adornados de testimonio coherente de ser y hacer en una sociedad gestora de esperanzas y un futuro esplendoroso y feliz" (CONGREGACIÓN RELIGIOSA..., Anuario, 2001, p. 13).

\section{4.- Etapas de los Primeros Cincuenta años del Colegio Santa Isabel de Hungría}

\section{a) Las Hermanas como Directoras del Colegio (1961- 1991)}

Entre los años 1961 y 1991, los primeros treinta años del Colegio, la dirección recayó en las Hermanas, aplicándose un sistema de nombramiento similar a cualquier cargo dentro de la Congregación Religiosa y, específicamente para el Colegio Santa Isabel de Hungría, se "... determina como primer requisito que sea profesora, que sea educadora, que posea capacidad de liderazgo, que tenga ciertos criterios, es decir, que cumpla con el perfil de lo que uno quiere, que tenga empatía con la gente" (MADARIAGA, 2008).

Las diez Hermanas que tuvieron la tarea de ejercer este cargo en el Colegio Santa Isabel de Hungría fueron designadas en su mayoría a través de este sistema, salvo dos o tres excepciones, fueron las siguientes: Hna. Paula Hernández (1961-1966), Hna. Trinidad Serey (1967-1968), Hna. Lina Torres Espinoza (1969-1971), Hna. Camila Mella Palma (19721973), Hna. Rosario Valdivia Contreras (1974-1978), Hna. Gladys Lagos Mora (1979- 
1982), Hna. Mercedes Muñoz Valdés (1983), Hna. Gabriela Bórquez Villamán (1984-1985), Hna. Bernarda Madariaga Urzúa (1986-1989) y Hna. Lucía Toledo Toledo (1990-1991).

En este período la Escuela no sólo recibía niños de familias pobres; en sus filas había toda clase de gente, había profesionales, gente de clase media. Lo admirable era ver como ellos apoyaban la existencia del nuevo Colegio y si algo hacían, se notaba que la gente "... estaba contenta, tan contenta" (MORENO, 2002). Esto último, evidencia que la Escuela ha iniciado sus actividades como una iniciativa para gente de escasos recursos; sin embargo, también atrajo a personas con mayor solvencia frente a la carencia de colegios que presentaba la Comuna en esa época. No obstante, el asombro mayor para las Hermanas fue justamente la recepción de los apoderados, “... gente tan buena de corazón, tan cariñosa, muy respetuosa con nosotras, valoraban todo lo que hacían las Hermanas, con todo lo que se hacía..." (MORENO, 2002).

Otro dato interesante, es que el Colegio también fue apadrinado por la Colonia Húngara. Al parecer se pidió el apoyo a la Embajada de Hungría para cooperar con el establecimiento, lo cual se concretó con la construcción de los monolitos de la entrada y ello obligó a izar cada lunes la bandera de Chile acompañada de la bandera de Hungría, como símbolo de este acuerdo y apoyo ${ }^{7}$.

La reciprocidad entre la Escuela y la Comunidad, el cariño entregado, el afecto sentido, hace evocar a la Madre Martina, una de las Hermanas presente en las vicisitudes de los primeros años, las características de esta relación al decir:

[...] yo encuentro que la gente nos quería mucho y nosotros queríamos a la gente. Hicimos una especie de familia dentro del Colegio, no era un Colegio donde ellos entraban y se iban. Siempre hubo una conversación, una atención, como era chiquitito. Así, a cada niño uno lo conocía, había ido a su casa, visitado el hogar, entonces era muy rico, muy bonito (PÁEZ, 2002).

Como ex alumna, Marisa Cerda recuerda entre sus profesoras a la Hna. Paula, a la Hna. Eufrosina, a la Hna. Myriam, a la Hna. Verónica, a la Hna. Cruz, a la Hna. Gladys, todos nombres de las profesoras que compartían la tarea de enseñar. El aprendizaje recibido se destacaba por la forma en que era entregado, como una especie de cuento, por lo tanto, significativamente muy rico y lo describe así: "Tenían libros, tenían el entorno, había naturaleza, había árboles, ellas usaban todo, era como estar en la casa; para enseñarnos a leer usaban todo el entorno y muchas cosas que estaban en el lugar, cosas que no se olvidan nunca" (CERDA, 2008).

Entre sus recuerdos, distingue algunas diferencias entre su primer año de estudios y los correspondientes hasta Sexta Preparatoria. De ser un grupo pequeño de niños se fue produciendo un aumento de la matrícula por nivel, cada año se incorporaban más alumnos provenientes de diversos sectores de la comuna de La Cisterna.

En opinión de la Hna. Eufrosina, es fundamental destacar que cada actividad buscaba el complemento de todo, es decir, lo deportivo, lo espiritual, lo religioso, lo académico, lo afectivo, en suma, todo. Una tarea absolutamente propia de ellas, en esos años, era orientar en la fe, por lo tanto, niños y niñas eran preparados en la catequesis para la Primera Comunión. En ello, recibían el apoyo de un capellán para celebrar la eucaristía y la autorización de la Parroquia Don Bosco. Considerando la preparación entregada por las Hermanas y la esmerada preocupación en estos aspectos, se logró generar una impronta o sello en el alumnado y en sus padres, gente de mucho respeto y muy creyentes. 
El 9 de enero de 1964, a través del Decreto N 110, se cambió el nombre a la Escuela; a partir de ese momento, se llamará Escuela Básica Particular $N^{o} 56$ Santa Isabel de Hungría.

El año 1968 fue un año especial, tocándole a la Hna. Lina Torres aplicar la Reforma Educacional del Presidente Eduardo Frei Montalva iniciada en 1965, reemplazando a la Educación Primaria por la Educación General Básica, aumentando los estudios de este nivel de seis a ocho años. Por lo tanto, hubo que incorporar el Séptimo Básico y al año subsiguiente el Octavo Básico; la tarea fue construir dos nuevas salas para estos nuevos niveles y nuevamente se produjo una actividad mancomunada con los apoderados y posibilitó el espacio necesario para ampliar la educación a siete años y luego a ocho. En forma paralela, se mantuvo el perfeccionamiento de años anteriores de las Hermanas y profesores, ya sea en la Federación de Institutos de Educación Particular Católica-FIDE-, en los cursos de verano o en la Universidad Católica de Valparaíso. Según la Hna. Lina, una de las tareas que más se promovió en esos años, especialmente por los acuerdos del Concilio Vaticano II, fue renovar la clase de Religión en los diferentes cursos de la enseñanza básica; sobre ello, la Hermana recuerda que: “... porque por la FIDE nosotras estábamos al tanto de los nuevos programas de Religión postconciliar. Como estábamos afiliadas a la FIDE primaria, allí se promovían estas ideas" (TORRES, 2002).

En relación a la Revista de Gimnasia que se comenzó a realizar todos los años, la Hermana Myriam rescata el hecho de que la organización pudiendo ser vertical, no lo era y señala: "Nosotras teníamos reuniones con la Comunidad y ahí conversábamos lo que se iba a hacer; nunca se hacía por la sola decisión de la Directora; todas lo acordábamos" (OBREQUE, 2003). La participación de las Religiosas, profesoras y comunidad escolar era fundamental.

Unas Hermana que asume la Dirección cuando el establecimiento ya contaba con alrededor de setecientos alumnos, divididos en dos niveles paralelos (A y B), recuerda de esa época:

[...] la gente era cariñosa, los profesores eran unidos, mantuvimos rara vez roces con ellos. Lo pasábamos estupendo, se hacían convivencias maravillosas, era todo maravilloso; realmente se trabajaba con amor, con gusto, porque los apoderados eran entusiastas y los profesores tenían una entrega generosa. Uno trabajaba harto y con amor, con harto cariño, uno amaba a los chiquillos, se inventaban cosas para hacer actividades; la cosa es que cada año fuera progresando un poquito más dentro de nuestros medios; porque realmente nosotras, las monjas, tenemos una característica $\mathrm{y}$ es que no somos comerciantes. Nosotros usamos nuestros medios e íbamos más allá. Era bonito, muy linda labor. (VALDIVIA, 2002).

Una ex funcionaria de ese período recuerda así a la Hna. Rosario:

[...] las hacía andar derechas. Trabajamos muy bien organizadas. Era una monjita que se relacionaba muy bien con los apoderados, con el Centro de 
Padres. En ese tiempo como que no había muchos adelantos como ahora, pero sí la parte académica estuvo muy bien. Hubo cosas muy bonitas, actividades, especialmente en la Básica... (SÁEZ, 2008).

El Colegio empezó a contar con la Unidad Técnico Pedagógica -UTP- con un profesor especialista como Jefe Técnico lo que favoreció las líneas de trabajo dando al profesor el ambiente adecuado para elaborar sus clases, tener la tranquilidad que le permitía laborar a gusto con la asesoría necesaria.

Sobre la Hna. Gladys, otra Directora, una profesora de la época y por varios años Presidenta del Sindicato del establecimiento, recuerda: “...Ella tenía una llegada muy especial con la gente, era muy afectuosa. Era como maestro chasquilla; se echaba a perder el equipo, ella lo arreglaba. Ella estaba en todas, siempre presente" (MANSILLA, 2008).

Nuevamente, el esfuerzo mancomunado entre las Hermanas, profesores y apoderados, coordinados por la Directora, Hna. Gladys, fue fundamental para encarar las nuevas exigencias y posibilitar la construcción de un nuevo pabellón para albergar a la Enseñanza Media. El proceso de incorporación de estos cursos fue paulatino, un año es el Primero Medio, al siguiente el Segundo Medio y así sucesivamente. Al iniciarse el Primer Año Medio cambió la modalidad impartida por el establecimiento, dejando de ser Colegio de Enseñanza Básica, integrándose la Enseñanza Media Científico-Humanista. Esta nueva realidad se estableció legalmente en la Resolución Exenta Nº 6.892 del 24 de junio de 1982, dictada por el Ministerio de Educación. Con ello también se eliminó su número como Escuela y será llamada en lo sucesivo Colegio Santa Isabel de Hungría.

Respecto a la preparación sacramental, la Hna. Mercedes señala que los apoderados debían acudir a la Parroquia donde los atendía el P. Carlos, s.d.b. El sistema era mediante fichas, que ellos estudiaban en forma paralela con los niños. A partir de sus recuerdos, la Hna. Mercedes destaca lo siguiente:

[...] El Padre Carlos les hablaba y decía que llamaba a los padres en especial, los llamaba los días domingo, quizás alguno no iría, pero la mayoría iba porque las mamás decían siempre que iban y los padres tenían que ir. Les decía 'miren en la ficha, vean esto y lo otro, aquí van los niños y ustedes'. Los hacía repasar. Tenían en la palma de la mano todo el panorama de sus hijos (MUÑOZ, 2002).

En el lapso de dos años a cargo de la Dirección, la Hna. Gabriela realizó una labor eficiente que permitió en su período el egreso de la primera generación de Cuarto Año Medio. No obstante, durante el ejercicio de su cargo tuvo en contra a los padres y apoderados y ellos se mantuvieron alejados del quehacer de las Hermanas; no se evidenciaba la unidad y el compromiso característico de generaciones previas. Sin embargo, Marisa Cerda, apoderada, guarda un especial recuerdo de la Hna. Gabriela, señala que “... era como muy tierna con los niños, era muy cercana a los cabros chicos y exigía a los apoderados, ya que si tenían sus hijos allí, había que preocuparse..." (CERDA, 2008).

De su período, la Hna. Bernarda señala que en su gestión el énfasis estuvo “...el carisma y la espiritualidad del Colegio. Es decir, es franciscano, porque se distinguía y porque surgió de los ideales de Nuestra Fundadora..., trabajamos a ese nivel..." (MADARIAGA, 2008).

La Hna. Bernarda se esforzó por unir a todos los estamentos del Colegio y revitalizar el compromiso algo perdido. Logró el apoyo del Centro de Padres y Apoderados, lo que se reflejó en una serie de actividades que se desarrollaron bajo su mandato, entre ellas la celebración de las Bodas de Plata (el 25 Aniversario) del Colegio Santa Isabel de Hungría. 
La última Directora religiosa, Hna. Lucía, no poseía la rigurosidad o exigencia de otras Hermanas en el cargo; ella dejaba hacer y generalmente decía sí a todo, iniciándose una crisis. En opinión de Marisa Cerda (2008), la crisis del Colegio y de la administración de las Hermanas se produjo a fines de los 80 . Se fundamenta en el hecho de que las Hermanas eran incapaces de sostener económicamente el Colegio, el cual había crecido en demasía y ya no era el pequeño colegio que ellas iniciaron. Ellas tenían y mantenían la idea de un Colegio pequeño formado como una familia.

\section{b) Administración de la Corporación Educacional del Arzobispado de Santiago (1992- 1997)}

Por la imposibilidad de las Hermanas para proseguir dirigiendo el Colegio fue cedido en Comodato al Arzobispado de Santiago y, la Corporación Educacional del mismo, asumió la dirección y coordinación del Colegio por un período de seis años, siendo el representante legal, el señor José Isla. Hubo dos Directores laicos: Flor Madariaga Allende (1992 - 1996) y José Raúl Toro Vallejos (1997).

En el ámbito pedagógico, se mantuvo la matrícula y en 1993, el Colegio adquirió la condición de Colegio Particular Subvencionado con Financiamiento Compartido y, de esta forma, pudo continuar con más recursos su servicio. En este aspecto tampoco se observaron cambios o adelantos evidentes, como se esperaba; más bien se acentúan ciertos mitos que Marisa Cerda manifiesta de la siguiente forma:

[...] el Colegio ha tenido buena fama en la Básica, daba muy buena base, no así la Media, se consideraba mala; por lo tanto, se iban al Instituto Nacional, al Liceo $\mathrm{N}^{\circ} 1$, al Carmela Carvajal8, y a otros. Claro, quedaban en ellos porque tenían buenas notas y estaban bien preparados, ellos se llevaban los aplausos, pero la formación de los niños pertenecía a las Hermanas (CERDA, 2008).

Por su parte, Marisa Cerda es enfática al plantear su visión sobre los avances de la época en cuanto a infraestructura. Su percepción es que no hubo ninguno y la razón es que el Arzobispado manifestará en el tiempo la posible adquisición del Colegio a las Hermanas; por lo tanto, les convenía su estado de poco avance para que fuera menos rentable y fácil de adquirir, en función de ello señala: “... fue una estrategia buena, que gracias a Dios no resultó y ellos trajeron muchos profesores nuevos, mucha gente venía con ellos. Fueron justamente aquellos que, cuando se fueron estos administradores, comenzaron a crear los problemas a las Hermanas..." (CERDA, 2008).

\section{c) Un Grupo de Laicos a Cargo de la Administración (1998-2011) ${ }^{9}$}

Los años en que el Colegio Santa Isabel de Hungría estuvo administrado por la Corporación Educacional dependiente del Arzobispado no logró consolidarse en el quehacer educativo, debido, en primer lugar, a que las Hermanas fueron prácticamente alejadas de esta tarea; en segundo lugar, la Corporación no realizó ningún esfuerzo por innovar la institución y revalidar su acción e influencia en la comuna de La Cisterna como se había logrado años antes; en tercer lugar, se formó un sindicato, el cual se politizó, lo cual tampoco favoreció el compromiso de los profesores por sacar adelante a la institución.

Ante ello, la Congregación, de común acuerdo con su Obispo, Mons. Manuel Camilo Vial, decidió suspender el Comodato y entregar en Administración directa a un grupo de 
laicos, encabezado por Jaime Caiceo Escudero ${ }^{10}$, a quien conocían desde su natal ciudad de Los Andes; sus estudios los realizó en el Instituto Chacabuco de los Hermanos Maristas de esa ciudad; su labor docente y directiva se ha plasmado en varias instituciones, entre ellas el colegio de los Sagrados Corazones de la Alameda, sede en los veranos de la FIDE Primaria para el curso de Perfeccionamiento para Directores, donde conoció en su calidad de docente a la Hna. Bernarda Madariaga y a la Hna. Rosario Valdivia, quienes asistían a esos cursos como alumnas. En 1996, su labor como académico de la Universidad Metropolitana de Ciencias de la Educación, lo llevó a convertirse en profesor guía de la tesis de grado de la Hna. Bernarda Madariaga para sacar su Magíster en Educación, Mención Orientación. En dicha ocasión, la Hna. Bernarda le dio a conocer la realidad que vivía el Colegio Santa Isabel de Hungría, naciendo así la posibilidad concreta de dirigir el establecimiento. La Superiora de la Congregación, Hna. Eufrosina Moreno y su Consejo, aceptaron la propuesta realizada por la Hna. Bernarda Madariaga y los nuevos laicos asumieron la conducción del establecimiento.

Ana María Carrizo, actual subdirectora, recuerda cómo estaba el Colegio en 1998: “... Había en el establecimiento un desorden administrativo en todas las instancias asesoras a la Dirección y que tenían que ser grupos de apoyo, pero en vez de ello, eran obstáculos..." (CARRIZO, 2008).

En opinión de Marisa Cerda, la llegada del nuevo equipo fue importante para preservar el Colegio; sin embargo, ella admite que tenía reticencias al respecto, pero al verlos actuar frente a los antiguos administradores, frente al sindicato y a todos en general, piensa que su llegada impuso el orden, se rescató el derecho de las Hermanas y se observó un mayor compromiso por sacar adelante al establecimiento, al cual ellas y otras personas se sumaron. Expresa, al respecto: “...en el fondo mi fin siempre ha sido defender a las monjas y a esta Dirección, porque si don Jaime lo hubiese hecho mal, no le quepa la menor duda que yo hubiese sido su peor enemiga..." (CERDA, 2008).

Pasado los momentos difíciles, de toma de posesión del nuevo equipo directivo y los despedidos que hubo al finalizar el primer año, los profesores antiguos junto con los que llegan se dan cuenta que había que sumarse al cambio de administración, porque su trabajo evidenciaba mayor seriedad. Para Ana María Carrizo no hay duda que esto se deriva de la acción y logros del Director, manifestando al respecto:

[...] yo creo que la gente confió en don Jaime como líder innato y confió también en su palabra. Hoy día no cabe la menor duda de que es una persona confiable y puede llevar a cabo los proyectos en forma rigurosa y ordenada, y que jamás va a prometer algo que no va a cumplir (CARRIZO, 2008).

En 1999 se dio inicio al proceso de mejorías y crecimiento del Colegio, se hizo necesario plantear metas de corto y largo plazo, tales como:

* Revisar el proyecto educativo.

* Mejorar la infraestructura.

* Fortalecer el clima laboral.

* Mejorar los rendimientos escolares en todos los ámbitos.

* Preparar la Jornada Escolar Completa.

Estas ideas serán las directrices que el Colegio Santa Isabel de Hungría debió asumir en los siguientes años para transformar a esta institución en un Colegio importante y de 
prestigio en La Cisterna, plasmándose en tres pilares básicos: Pastoral, Académico e Infraestructura.

En los más de 18 años que dirige el Colegio Santa Isabel de Hungría la actual Dirección se han logrado significativos avances en el SIMCE, tanto en los cuartos y octavos básicos como en los segundos medios, subiendo los puntajes desde un promedio de 242 puntos a 280 puntos en los cuatro sectores involucrados: lenguaje, matemática, ciencias sociales y ciencias naturales.

De estos resultados dan fe las personas que han visto como el Colegio ha dejado atrás los años del comodato con la Corporación Educacional del Arzobispado de Santiago. Marisa Cerda señala:

[...] han hecho muchas cosas en este Colegio; ahora es otra cosa. Por ejemplo, la cantidad de alumnos, hay mucha gente en este Colegio, es barato, tiene bonita infraestructura, tiene buena educación, de hecho la excelencia no es una cuestión que sea algo que se adjudica, sino que viene de los resultados y éstos son índices que mide el Ministerio, en eso no se puede mentir... (CERDA, 2008).

En el año 2010, el Colegio alcanzó una matrícula de mil ciento setenta alumnos que no sólo provienen de La Cisterna, sino que de comunas aledañas ${ }^{11}$. En opinión de Gladis Meli, secretaria desde el primer período de la historia del colegio, observar sus salas amplias, luminosas, espacios libres, medios audiovisuales, la existencia de preuniversitarios para los alumnos, la preparación para el SIMCE, en todo se nota planificación estratégica. Ella expresa:

[...] es un personal más idóneo, hay profesores y profesoras. Yo veo que se supervisa más los contenidos, hay más preocupación para que los chicos avancen. Esto yo no lo veía antes... Ahora me doy cuenta porque lo veo más, sé de qué se trata $\mathrm{y}$, como soy mamá con dos hijas en el establecimiento, lo valoro más; eso debe ser, cambia la visión (MELI, 2008).

La festividad del 4 de octubre posee un tinte ecológico debido a que San Francisco es Patrono de la Naturaleza. Según la Hna. Graciela Quiroz, se quiere que “... los niños conozcan quién fue San Francisco, todo de él y una de las cosas es que él amaba la naturaleza. Entonces, los chicos aprenden todo lo que se plantea al respecto, todo lo que son las flores, que todo lo natural hay que cuidarlo..." (QUIROZ, 2009). Hoy con el cambio climático, esta formación, cariño y aprecio por la naturaleza es fundamental.

\section{Conclusiones}

Se ha entregado, a partir especialmente de testimonios orales, la Historia del Cincuentenario del Colegio Santa Isabel de Hungría. Para el futuro, es necesario: (a) Continuar en la senda de la Pastoral de los últimos años; educar, entregando valores propios del humanismo cristiano, como objetivo transversal básico, es el gran desafío del presente y del futuro. (b) La educación católica analizada en el tiempo es sinónimo de calidad. El Colegio Santa Isabel de Hungría no puede estar ajeno a ello. (c) Se debe conservar el clima 
de convivencia sana y armónica alcanzado en los últimos años, lo cual es resaltado por los padres y apoderados, alumnos y personal del Colegio. (d) Conservar el número de alumnos, cifra que alcanza los 1.193 entre niños, niñas y jóvenes, a pesar de los índices bajos de natalidad. (e) Proseguir afianzando la estrecha colaboración que los Padres y Apoderados han expresado en su Centro, que siempre tuvieron en el pasado y mantienen en el presente. (f) La infraestructura ha tenido importantes cambios en los últimos años; sin embargo, quedan aún tareas por realizar.

Mantener las buenas relaciones y la confianza mutua entre las Hermanas y la actual administración es crucial. La gestión realizada apunta a preservar la obra que ellas iniciaron, lo que ha dado origen a una estrecha unión donde se cumplen las expectativas del grupo administrador, de ellas y de su Madre Fundadora. El lema de la actual Dirección es lograr que el Colegio Santa Isabel de Hungría se transforme en el mejor de la comuna de La Cisterna; ése es el desafío y la meta a mediano plazo.

\section{Fuentes de Consulta}

\section{a) Libros}

CAICEO, Jaime. Huellas de Amor de una Mujer Chilena: Congregación Religiosa Hermanas Franciscanas Cooperadoras Parroquiales-Colegio Santa Isabel de Hungría. Santiago de Chile: Imprenta Madrid, 2010.

CAICEO, Jaime et al. Educación Rural en Iberoamérica. Madrid: Anroart Ediciones, 2009.

SOCÍAS, Estela. Un Camino a la Santidad: La Vida de la Madre Teresa Ortúzar Ovalle. Santiago de Chile: Imprenta Madrid, 2010. Disponible em: 〈www.obispadoSanFelipe.cl>.

\section{b) Documentos}

CONGREGACIÓN RELIGIOSA HERMANAS FRANCISCANAS COOPERADORAS PARROQUIALES (s/a). Biografía de la Madre Teresa Ortúzar Ovalle. Los Andes: s/e.

CONGREGACIÓN RELIGIOSA HERMANAS FRANCISCANAS COOPERADORAS PARROQUIALES (s/a). Breve Historia de las Hermanas Franciscanas Cooperadoras Parroquiales. Los Andes: s/e.

CONGREGACIÓN RELIGIOSA HERMANAS FRANCISCANAS COOPERADORAS PARROQUIALES (s/a). Naturaleza, Espiritualidad y Carisma Fundacional (Incluye breve reseña histórica de los fundadores). Los Andes: Instituto Diocesano.

CONGREGACIÓN RELIGIOSA HERMANAS FRANCISCANAS COOPERADORAS PARROQUIALES, COLEGIO SANTA ISABEL DE HUNGRÍA. Proyecto Educativo Institucional. Santiago de Chile: Imprenta Clavel, 2002.

MADARIAGA, Hermana Bernarda. Espiritualidad de la Madre Teresa Ortúzar Ovalle, Fundadora de las Hermanas Franciscanas Cooperadoras Parroquiales Chilenas. Los Andes: 1993.

c) Anuario 
CONGREGACIÓN RELIGIOSA HERMANAS FRANCISCANAS COOPERADORAS PARROQUIALES (2000-2010). Colegio Santa Isabel de Hungría. Anuario. Santiago de

Chile: Imprenta Madrid Ltda, 2000-2010 (11 ediciones diferentes, una por año).

\section{d) Testimonios Orales}

CAICEO, Jaime (2008). 60 años, Director del Colegio Santa Isabel de Hungría, La Cisterna, 4 de agosto.

CARRIZO, Ana María (2008). 55 años, Sub-Directora del Colegio Santa Isabel de Hungría, La Cisterna, 4 de octubre.

CERDA, María Eloísa (2008). 53 años, exalumna y ex Presidenta del Centro de Padres del Colegio Santa Isabel de Hungría, La Cisterna, 21 de enero.

FUENTES, Vicente (2008). 52 años, Inspector General del Colegio Santa Isabel de Hungría, La Cisterna, 31 de julio.

IBARRA, Claudia (2008). 21 años, exalumna y ex Presidenta del Centro de Alumnos del Colegio Santa Isabel de Hungría, La Cisterna, 25 de enero.

MADARIAGA, Bernarda, hfcp (2008). 52 años, Superiora General de la Congregación Religiosa Hermanas Franciscanas, La Cisterna, 13 de marzo.

MANSILLA, Celeste (2008). 53 años, Presidenta del Sindicato del Colegio Santa Isabel de Hungría, La Cisterna, 7 de agosto.

MELI, Gladys (2008). 45 años, Secretaria del Colegio Santa Isabel de Hungría, La Cisterna, 18 de enero.

MORENO, Eufrosina, hfcp (2002). 67 años, ex Superiora General de la Congregación Religiosa Hermanas Franciscanas, Los Andes, 25 de julio.

MUÑOZ, Mercedes, hfcp (2002). 74 años, Los Andes, 24 de julio.

OBREQUE, Myriam, hfcp (2003). 64 años, Los Andes, 25 de enero.

PÁEZ, Martina, hfcp (2002). 70 años, ex Superiora General de la Congregación Religiosa Hermanas Franciscanas, Los Andes, 25 de julio.

QUIROZ, Graciela, hfcp (2009). 50 años, La Cisterna, 9 de marzo.

SÁEZ, Sabina (2008). 71 años, ex Funcionaria del Colegio Santa Isabel de Hungría, La Cisterna, 18 de enero.

TORRES, Lina, hfcp (2002). 75 años, La Cisterna, 24 de julio.

VALDIVIA, Rosario, hfcp (2002). 58 años, Los Andes, 25 de julio. 
YUFFER, Tatiana (2008). 67 años, ex Profesora del Colegio Santa Isabel de Hungría, La Cisterna, 25 de julio.

\section{Notas}

${ }^{1}$ Este trabajo se realizó a partir de una investigación realizada a propósito del cincuentenario de la fundación de este establecimiento educacional.

2 Académico de la Universidad de Santiago de Chile, Director del Colegio Santa Isabel de Hungría y Miembro del Directorio de la Sociedad Chilena de Historia de la Educación; correo: jcaiceo@ hotmail.com

${ }^{3}$ En Chile se nombra por Escuela al establecimiento educacional que sólo tiene enseñanza primaria o básica.

${ }^{4}$ En Chile se nombra por Colegio al establecimiento educacional que posee enseñanza primaria o básica y secundaria o media.

${ }^{5}$ In Chile "escuela" is named for an institution that has only primary education.

${ }^{6}$ In Chile the name "colegio" is named for an educational institution that has primary and secondary education.

${ }^{7}$ En el 2010, volvió a aparecer la Embajada apoyando al establecimiento en actividades culturales y con la construcción de una efigie de Santa Isabel de Hungría que está ubicada en el patio principal.

${ }^{8}$ Establecimientos públicos emblemáticos, es decir, de alta exigencia y calidad.

${ }^{9}$ Este mismo equipo dirige el establecimiento educacional hasta el momento ininterrumpidamente (2017).

${ }^{10}$ Profesor de Filosofía y Doctor en Ciencias de la Educación, quien comenzó a ejercer los roles de Representante Legal y Director, secundado por Ana María Carrizo Gutiérrez, Educadora de Párvulos y Magíster en Educación y Sofía Bustos Larenas, Contador Auditor.

${ }^{11}$ En el 2016 se alcanzó la cantidad de 1193 alumnas y alumnos.

Recebido: Abril/2017.

Aprovado: Junho/2017. 